\title{
xrdfit: A Python package for fitting synchrotron X-ray diffraction spectra
}

\section{Peter Crowther ${ }^{1}$ and Christopher S. Daniel ${ }^{1}$}

DOI: $10.21105 /$ joss. 02381

\section{Software \\ - Review ¿ \\ - Repository ¿ \\ - Archive ct}

Editor: Jeff Gostick [

\section{Reviewers:}

- QKedoKudo

- @mikapfl

Submitted: 22 May 2020 Published: 31 August 2020

\section{License}

Authors of papers retain copyright and release the work under a Creative Commons Attribution 4.0 International License (CC BY 4.0).
1 The University of Manchester, UK

\section{Summary}

The evolution of peak profiles in synchrotron X-ray diffraction (SXRD) data can tell us how the internal crystallographic structures of metals change during applied heating, high temperature straining and cooling cycles (Canelo-Yubero et al., 2016; Daniel, Nguyen, Atkinson, \& Quinta da Fonseca, 2019; Hu, Sun, Hector, \& Ren, 2017; Stark et al., 2015), which is invaluable information used to improve industrial processing routes (Salem, Glavicic, \& Semiatin, 2008). The experiment requires a beamline, at a synchrotron radiation facility such as Diamond Light Source (Diamond Light Source Ltd, 2020), to produce a high energy X-ray beam and illuminate a polycrystalline sample (Daniel et al., 2019). The results are recorded in the form of timeresolved diffraction pattern rings, which are converted into a spectra of intensity peaks versus two-theta angle for a given direction (Ashiotis et al., 2015; Filik et al., 2017; Hammersley, Svensson, Hanfland, Fitch, \& Hausermann, 1996). However, since many intensity profiles are collected during each experiment, with detectors recording at speeds greater than $250 \mathrm{~Hz}$ (DECTRIS - detecting the future, 2020; Loeliger et al., 2012), fitting each of the individual lattice plane peaks can take a long time using current available software (Basham et al., 2015; Hammersley, 2016; Merkel \& Hilairet, 2015).

There are existing packages which can be used to fit peaks in SXRD spectra, examples include DAWN (Basham et al., 2015), Multifit/Polydefix (Merkel \& Hilairet, 2015) and Fit2d (Hammersley, 2016). In these cases, the software are compiled packages with a graphical user interface. Setting up the peak fits usually involves a point and click method to select the peak bounds, meaning it is unlikely to create a repeatable analysis. These packages also struggle to distinguish any peaks that overlap, which is important for capturing changes in multiphase materials (Daniel et al., 2019). MAUD (Lutterotti, Vasin, \& Wenk, 2014) is a software package that approaches fitting in a different way. MAUD uses the Rietveld refinement method (Rietveld, 1969) to match a model of the beamline setup and material properties to the data. This method allows determination of additional material properties, such as crystallographic texture, but applies an averaging over the peak positions and intensities to fit the model, meaning individual peak shifts cannot be accurately determined.

xrdfit is a Python package we have developed for a faster fitting of diffraction peaks in SXRD (and XRD) spectra, which can be used for datasets containing many thousands of patterns. It is intended as an easy to use tool which enables automated, repeatable identification of peak positions and profiles in spectra with multiple individual or overlapping lattice plane peaks. The features of xrdfit are shown schematically in Figure 1. xrdfit uses the Python package lmfit (Newville, Stensitzki, Allen, \& Ingargiola, 2014) for the underlying fitting. Features are included for selecting different 'cakes' of data and automating fitting over many spectra, to enable tracking of peaks as they shift throughout the experiment. By analysing how different lattice plane peaks change during simulated processing, as can be seen in Figure 2, the transformation and micromechanical behaviour of the material can be understood. 
a)
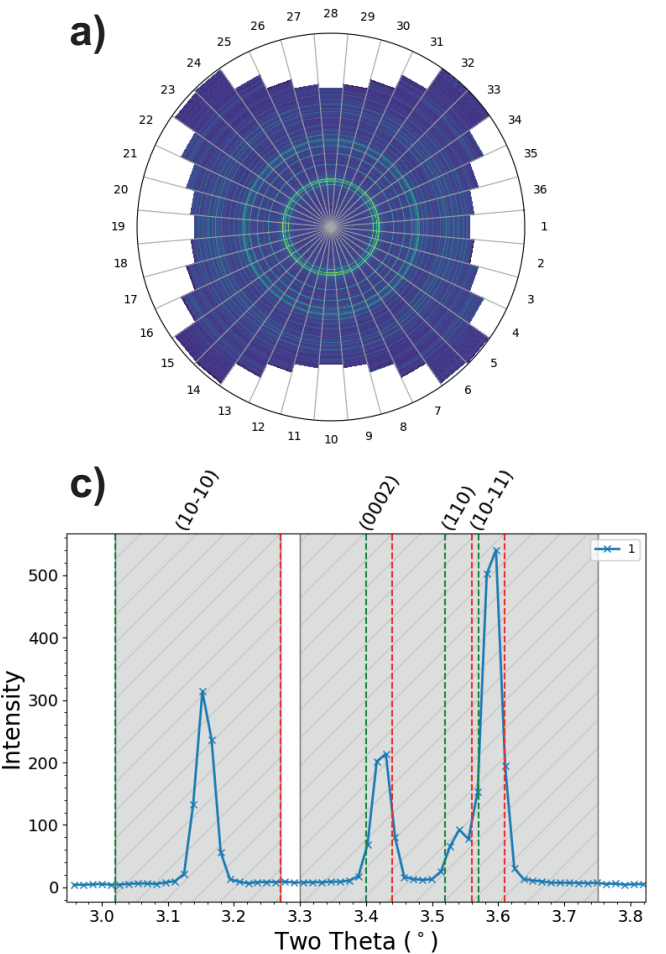

b)

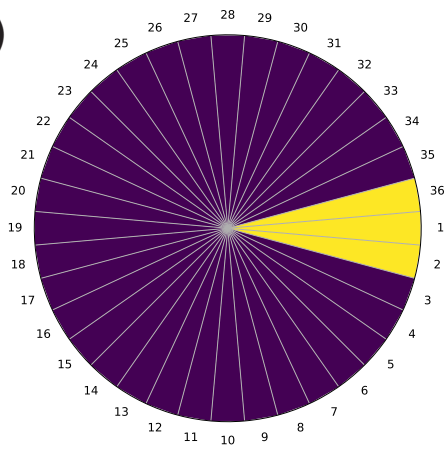

d)

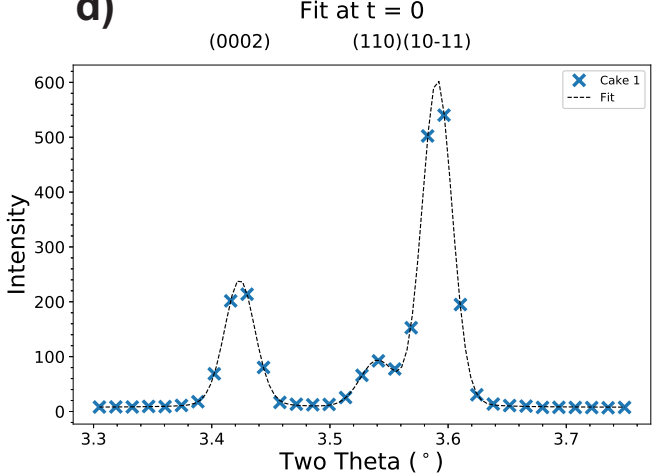

Figure 1: A schematic representing the features of xrdfit and the analysis of a synchrotron X-ray diffraction (SXRD) pattern, showing: (a) a polar plot of the caked intensity data; (b) an option for selecting different cakes and merging caked datasets; (c) adjustable peak bounds (grey) and adjustable maxima and minima bounds (red and green) for constraining the peak fit; (d) an example fit of multiple and overlapping peaks.

xrdfit is designed to be accessible for all researchers who need to process SXRD (and XRD) spectra and so does not require a detailed knowledge of programming or fitting. The package has been used for the analysis of data taken during the hot deformation of a two-phase titanium alloy, which is presented in an article currently in press (Daniel et al., 2019), and will be used for future studies investigating the high temperature processing of metals in our research group. We hope that its public release will allow other researchers to benefit from fast data fitting (up to 40 times faster than the current software), reducing the effort required to analyse their experimental data. 


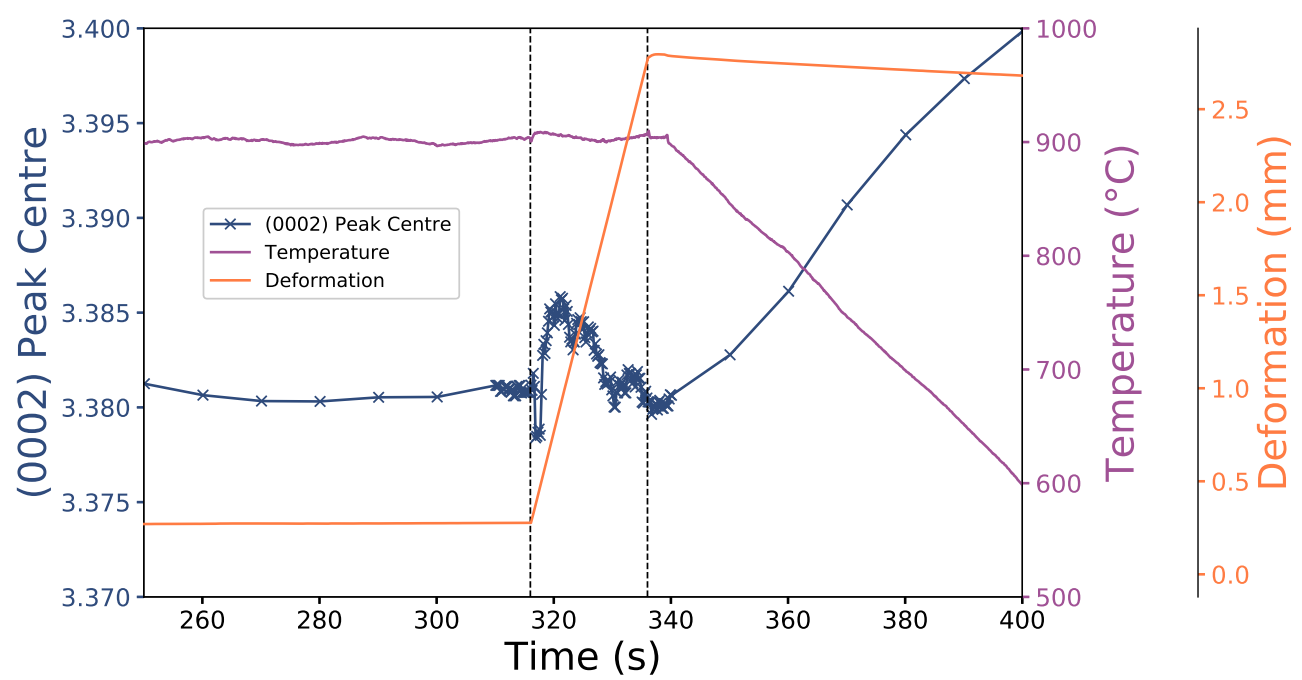

Figure 2: An example analysis of a two-phase titanium (Ti-6Al-4V) alloy during high temperature and high strain rate deformation, showing characteristic shifts of the $\alpha(0002)$ peak centre. The shifts of the peak can be used to calculate elastic lattice straining in the hexagonal close-packed (hcp) lattice, as well as measure the thermal contraction on cooling.

\section{Acknowledgements}

We acknowledge funding from the UK's Engineering and Physical Sciences Research Council (EPSRC) via LightForm (EP/R001715/1). We thank Oliver Buxton for his comments on a pre-release version of xrdfit.

\section{References}

Ashiotis, G., Deschildre, A., Nawaz, Z., Wright, J. P., Karkoulis, D., Picca, F. E., \& Kieffer, J. (2015). The fast azimuthal integration python library:pyFAl. Journal of Applied Crystallography, 48(2), 510-519. doi:10.1107/s1600576715004306

Basham, M., Filik, J., Wharmby, M. T., Chang, P. C. Y., Kassaby, B. E., Gerring, M., Aishima, J., et al. (2015). Data analysis WorkbeNch (DAWN). Journal of Synchrotron Radiation, 22(3), 853-858. doi:10.1107/s1600577515002283

Canelo-Yubero, D., Requena, G., Sket, F., Poletti, C., Warchomicka, F., Daniels, J., Schell, N., et al. (2016). Load partition and microstructural evolution during in situ hot deformation of ti6Al6V2Sn alloys. Materials Science and Engineering: A, 657, 244-258. doi:10.1016/ j.msea.2016.01.059

Daniel, C. S., Nguyen, C.-T., Atkinson, M. D., \& Quinta da Fonseca, J. (2019). Direct evidence for a dynamic phase transformation during high temperature deformation in ti-64 [preprint]. Retrieved from https://doi.org/10.5281/zenodo.3381183

DECTRIS - detecting the future. (2020). PILATUS3 X CdTe 2M. Retrieved from https:// www.dectris.com/products/pilatus3/pilatus3-x-cdte-for-synchrotron/pilatus3-x-cdte- $2 \mathrm{~m}$ /

Diamond Light Source Ltd. (2020). Diamond Light Source. Retrieved from https://www. diamond.ac.uk 
Filik, J., Ashton, A. W., Chang, P. C. Y., Chater, P. A., Day, S. J., Drakopoulos, M., Gerring, M. W., et al. (2017). Processing two-dimensional x-ray diffraction and smallangle scattering data inDAWN 2. Journal of Applied Crystallography, 50(3), 959-966. doi:10.1107/s1600576717004708

Hammersley, A. P. (2016). FIT2D: A multi-purpose data reduction, analysis and visualization program. Journal of Applied Crystallography, 49(2), 646-652. doi:10.1107/ S1600576716000455

Hammersley, A. P., Svensson, S. O., Hanfland, M., Fitch, A. N., \& Hausermann, D. (1996). Two-dimensional detector software: From real detector to idealised image or two-theta scan. High Pressure Research, 14(4-6), 235-248. doi:10.1080/08957959608201408

Hu, X. H., Sun, X., Hector, L. G., \& Ren, Y. (2017). Individual phase constitutive properties of a TRIP-assisted QP980 steel from a combined synchrotron x-ray diffraction and crystal plasticity approach. Acta Materialia, 132, 230-244. doi:10.1016/j.actamat.2017.04.028

Loeliger, T., Brönnimann, C., Donath, T., Schneebeli, M., Schnyder, R., \& Trüb, P. (2012). The new pilatus3 asic with instant retrigger capability. In 2012 ieee nuclear science symposium and medical imaging conference record (nss/mic) (pp. 610-615). doi:10.1109/ NSSMIC.2012.6551180

Lutterotti, L., Vasin, R., \& Wenk, H.-R. (2014). Rietveld texture analysis from synchrotron diffraction images. I. Calibration and basic analysis. Powder Diffraction, 29(01), 76-84. doi:10.1017/S0885715613001346

Merkel, S., \& Hilairet, N. (2015). Multifit/polydefix: A framework for the analysis of polycrystal deformation using x-rays. Journal of Applied Crystallography, 48(4), 1307-1313. doi: $10.1107 /$ s 1600576715010390

Newville, M., Stensitzki, T., Allen, D. B., \& Ingargiola, A. (2014). LMFIT: Non-linear leastsquare minimization and curve-fitting for python. Zenodo. doi:10.5281/ZENODO.11813

Rietveld, H. M. (1969). A profile refinement method for nuclear and magnetic structures. Journal of Applied Crystallography, 2(2), 65-71. doi:10.1107/S0021889869006558

Salem, A. A., Glavicic, M. G., \& Semiatin, S. L. (2008). The effect of preheat temperature and inter-pass reheating on microstructure and texture evolution during hot rolling of ti6AI4V. Materials Science and Engineering: A, 496(1-2), 169-176. doi:10.1016/j.msea.2008.05. 017

Stark, A., Rackel, M., Tankoua, A. T., Oehring, M., Schell, N., Lottermoser, L., Schreyer, A., et al. (2015). In situ high-energy x-ray diffraction during hot-forming of a multiphase TiAl alloy. Metals, 5(4), 2252-2265. doi:10.3390/met5042252 